\title{
Estimativas volumétricas em povoamento de Pinus caribaea var. hondurensis no sudoeste da Bahia
}

\author{
Lara Clímaco de Melo¹, Patrícia Anjos Bittencourt Barreto², Francisco Garcia Romeiro Barbosa de Oliveira ${ }^{3}$, Adalberto Brito de \\ Novaes $^{2}$
}

${ }^{1}$ Universidade Federal do Paraná, Av. Lothário Meissner, 900, Jardim Botânico, CEP 80210-170, Curitiba, PR, Brasil.

2Universidade Estadual do Sudoeste da Bahia, Estrada do Bem Querer, CEP 45083-900, Vitória da Conquista, BA, Brasil

${ }^{3}$ Projeto Mata Branca, CAR-SEDIR (BA), Rua 6 de outubro, CEP 46620-000, Contendas do Sincorá, BA, Brasil.

*Autor correspondente:

laracmelo@gmail.com

Termos para indexação:

Modelos volumétricos

Fator de forma

Quociente de forma

Index terms:

Volumetric models

Form factor

Form quotient

Histórico do artigo:

Recebido em 04/12/2012

Aprovado em 25/11/2013

Publicado em 31/12/2013
Resumo - Objetivou-se com este trabalho selecionar modelos volumétricos e avaliar a eficiência de três métodos para estimativa de volume de madeira para Pinus caribaea em povoamento equiâneo no município de Vitória da Conquista, BA. Foram selecionadas, abatidas e cubadas rigorosamente árvores para obtenção do volume individual total e comercial, com casca e sem casca. Foram ajustados oito modelos volumétricos. Os modelos de maior desempenho foram selecionados com base no valor ponderado dos escores dos parâmetros estatísticos. Os volumes obtidos com a cubagem foram comparados aos obtidos por meio do fator de forma, quociente de forma e modelos ajustados. O modelo de Stoate foi o mais eficiente na estimativa dos volumes com casca total e comercial. Os modelos de Stoate e de Naslund modificado apresentaram maior desempenho para estimar os volumes sem casca total e comercial, respectivamente. $\mathrm{O}$ fator de forma e os modelos volumétricos são recomendados para a predição com precisão do volume de madeira da espécie. doi: 10.4336/2013.pfb.33.76.459

\section{Volumetric estimates in a stand of Pinus caribaea var. hondurensis in Bahia State, Brazil}

\begin{abstract}
The objective of this work was to select volumetric models and evaluate the efficiency of three methods to estimate the wood volume of Pinus caribaea in an evenaged stand in the city of Vitória da Conquista, BA. Trees were selected, felled and their cubage was rigorously measured for total individual and commercial volume, with and without bark. Eight volumetric models were adjusted to the volumes. Higher performance models were selected based on the weighted value of the statistical parameters scores. The volumes obtained with cubage measurement were compared to those obtained by form factor, form quotient and adjusted models. The Stoate model was the most efficient in estimating total and commercial volumes with bark. The Stoate model and the modified Naslund model had better performance in estimating the total and commercial volumes, respectively, without bark. The form factor and the volumetric models are recommended for accurately predicting wood volume for this species.
\end{abstract}




\section{Introdução}

No Brasil, as plantações florestais ocupam atualmente cerca de 6,5 milhões de ha. Destes, 1,8 milhões correspondem ao gênero Pinus. No Estado da Bahia, em particular, estima-se que o gênero ocupa uma área de 21.520 ha (Anuário..., 2012).

A introdução de diferentes espécies tornou o plantio de pinus viável em todo Brasil, constituindo uma importante fonte de madeira para diversos fins, como para indústria de celulose, lâminas e chapas, madeira serrada, compensados, energia, entre outros (Shimizu \& Medrado, 2005). O sucesso do seu estabelecimento pode ser atribuído à ampla variação de condições ambientais em sua distribuição original, que propiciou alta variabilidade genética e, como consequência, a adaptação do gênero a diversas condições ecológicas (Shimizu, 2006). Dentre as espécies de pinus mais cultivadas no país está o Pinus caribaea, que engloba três variedades, dentre as quais a hondurensis, uma das mais plantadas no mundo.

A expansão dos plantios comerciais de florestas no país sinaliza a crescente participação do setor florestal brasileiro, com a alta demanda por recursos energéticos e naturais. Por outro lado, representa a diminuição da pressão sobre os remanescentes florestais por abastecer o mercado antes atendido por espécies nativas, favorecendo a conservação da biodiversidade, proteção da água e o equilíbrio do clima (BRACELPA, 2013). Diante disso, o conhecimento do estoque madeireiro e potencial produtivo desses povoamentos torna-se fundamental para o correto manejo, planejamento e sustentabilidade da produção.

A predição do volume constitui uma das informações de maior importância para o diagnóstico do potencial de uma floresta. Vários métodos foram desenvolvidos para determinação dessa variável, entre eles estão o emprego do fator de forma e de equações volumétricas, normalmente ajustadas a partir de medições de diâmetro à $1,30 \mathrm{~m}$ do solo (DAP) e altura total das árvores.

Diversos modelos volumétricos foram testados para o gênero Pinus em diferentes regiões do Brasil (Machado et al., 2002, 2005; Moraes Neto, 2009; Pelissari et al., 2011; Téo et al., 2011). No entanto, são inexistentes aqueles que descrevem a volumetria para esse gênero no Estado da Bahia.

Assim, objetiva-se com esse trabalho: (a) selecionar modelos volumétricos de melhor ajuste e precisão e (b) avaliar a eficiência de três métodos para estimativa de volume de madeira para Pinus caribeae var. hondurensis, visando a destinação do produto para fins energéticos.

\section{Material e métodos}

\section{Área de estudo}

Os dados empregados nesse trabalho foram obtidos de um povoamento de Pinus caribaea var. hondurensis, com 10 anos de idade e espaçamento de $3 \mathrm{~m}$ x $3 \mathrm{~m}$, localizado na área experimental da Universidade Estadual do Sudoeste da Bahia - UESB, em Vitória da Conquista, BA, cujas coordenadas geográficas são $14^{\circ} 53^{\prime}$ 'S e $40^{\circ} 48^{\prime}$ W.

A região apresenta clima tropical de altitude $(\mathrm{Cwb})$, segundo classificação de Köppen, precipitação média anual de $733,9 \mathrm{~mm}$, altitude de $928 \mathrm{~m}$ e temperaturas médias de $25,3{ }^{\circ} \mathrm{C}$ (máxima) e de $16,1^{\circ} \mathrm{C}$ (mínima). O solo pertence à classe Latossolo Amarelo Distrófico (Superintendência de Estudos Econômicos e Sociais da Bahia, 2010).

\section{Obtenção dos dados}

Foram selecionadas e abatidas 30 árvores, distribuídas uniformemente em cinco classes diamétricas, com amplitude de $4 \mathrm{~cm}$, conforme Tabela 1 . O número de árvores-amostras utilizadas foi definido com base em estudos da literatura relacionada, que adotaram número amostral entre 10 e 40 árvores (Rios \& Mori, 2002; Ferrari et al., 2005; Moraes Neto, 2009; Soares et al., 2010; Azevedo et al. 2011; Pelissari et al., 2011).

A cubagem das árvores foi realizada por meio do método de Smalian. Os diâmetros com casca e sem casca foram medidos ao longo do fuste nas posições $0,3 \mathrm{~m}, 0,7 \mathrm{~m}, 1,3 \mathrm{~m}$ e a partir desse ponto as seções foram medidas de $2 \mathrm{~m}$ em $2 \mathrm{~m}$ até a posição onde ocorria diâmetro com casca de $4 \mathrm{~cm}$, sendo então medido o comprimento da ponta. O diâmetro comercial mínimo de $4 \mathrm{~cm}$ foi determinado com base no objetivo da produção, que será destinada para fins energéticos.

Tabela 1. Distribuição diamétrica das árvores de Pinus caribaea var. hondurensis.

\begin{tabular}{ccc}
\hline Classes de diâmetro (cm) & $\begin{array}{c}\text { Centro de classe } \\
(\mathbf{c m})\end{array}$ & Frequência \\
\hline $5-8,99$ & 9,5 & 10 \\
$9-12,99$ & 15,5 & 42 \\
$13-16,99$ & 21,5 & 108 \\
$17-20,99$ & 27,5 & 79 \\
$21-24,99$ & 33,5 & 7 \\
\hline
\end{tabular}


Os diâmetros das seções e as alturas das árvores cubadas foram medidos com suta e trena, respectivamente. De posse dos dados, foi calculado o volume com e sem casca de cada seção. Com o somatório dos volumes das seções, calculou-se o volume total e comercial, incluindo e excluindo o volume da ponta, respectivamente.

\section{Modelos volumétricos}

$\mathrm{O}$ ajuste de equações volumétricas foi utilizado para a predição dos volumes totais e comerciais das árvores com e sem casca. Foram avaliados 8 modelos descritos na literatura (Tabela 2), que apresentam como variável independente o volume (V) em função das variáveis dependentes DAP e/ou altura total da árvore $(\mathrm{Ht})$

Tabela 2. Modelos alométricos testados para estimativa dos volumes.

\begin{tabular}{lll}
\hline $\mathbf{N}^{\mathbf{o}}$ & \multicolumn{1}{c}{ Autor } & \multicolumn{1}{c}{ Modelo } \\
\hline 1 & Hohenadl-Kren & $\mathrm{V}=\beta_{0}+\beta_{1} \mathrm{~d}+\beta_{2} \mathrm{~d}^{2}$ \\
2 & Spurr & $\mathrm{V}=\beta_{0}+\beta_{1} \mathrm{~d}^{2} \mathrm{~h}$ \\
3 & Schumacher \& Hall log & $\mathrm{Ln} \mathrm{V}=\beta_{0}+\beta_{1} \operatorname{lnd}+\beta_{2} \operatorname{lnh}$ \\
4 & Stoate & $\mathrm{V}=\beta_{0}+\beta_{1} \mathrm{~h}+\beta_{2} \mathrm{~d}^{2}+\beta_{3} \mathrm{~d}^{2} \mathrm{~h}$ \\
5 & Näslund modificado & $\mathrm{V}=\beta_{0}+\beta_{1} \mathrm{~d}^{2}+\beta_{2} \ln \mathrm{d}^{2} \mathrm{~h}+\beta_{3} \mathrm{dh}^{2}+\beta_{4} \mathrm{~h}^{2}$ \\
6 & Equação linear & $\mathrm{V}=\beta_{0}+\beta_{1} \mathrm{~d}$ \\
7 & Koperzky\&Gehrhardt & $\mathrm{V}=\beta_{0}+\beta_{1} \mathrm{~d}^{2}$ \\
8 & Schumacher \& Hall & $\mathrm{V}=\beta_{0}+\beta_{1} \mathrm{~d}+\beta_{2} \mathrm{~h}$ \\
\hline
\end{tabular}

*V $=$ volume $\left(\mathrm{m}^{3}\right) ; \mathrm{d}=$ diâmetro a 1,30 $\mathrm{m}$ do solo $(\mathrm{cm}) ; \mathrm{h}=$ altura total $(\mathrm{m})$; $\mathrm{Ln}=\operatorname{logaritmo}$ neperiano; $\beta_{\mathrm{n}}=$ coeficientes de regressão.

Os melhores modelos foram selecionados com base nas seguintes estatísticas: coeficiente de determinação ajustado $\left(\mathrm{R}^{2}{ }_{\mathrm{aj}}\right)$, erro padrão da estimativa em sua forma absoluta e percentual $\left(\mathrm{S}_{\mathrm{yx}}\right)$, valor de $\mathrm{F}$, valor ponderado dos escores estatísticos (VP) e análise gráfica dos resíduos.

No modelo de Schumacher \& Hall log, em que a variável dependente sofreu uma transformação logarítimica, o erro padrão foi corrigido na escala original, para possibilitar a comparação com os modelos aritméticos. Desse modo, multiplicou-se o volume estimado pelo fator de correção de Meyer (1) para então recalcular o Syx, utilizando a fórmula (2).

$$
\text { Fator de Meyer }=e^{0,5 \cdot S_{y x}^{2}}
$$

Em que: $\mathrm{e}=2,718281828 ; \mathrm{S}_{\mathrm{yx}}=$ erro padrão da estimativa $\left(\mathrm{m}^{3}\right)$.

$$
S_{y x} \text { recalc }=\sqrt{\frac{\sum_{i=1}^{n}(y i-\hat{y} i)^{2}}{(n-p)}}
$$

Em que: $\mathrm{S}_{\mathrm{yx} \text { recalc }}=$ erro padrão da estimativa recalculado; yi $=$ valor real de cada observação; $\hat{Y} \mathrm{i}=$ valor estimado de cada observação; $\mathrm{n}=$ número de dados; $\mathrm{p}=$ número de coeficientes de cada equação.

O VP de cada modelo testado foi determinado conforme metodologia proposta por Thiersch (1997), a partir da atribuição de pesos para todos os parâmetros estatísticos considerados. Peso 1 para a equação mais eficiente, 2 para a segunda e assim sucessivamente. Posteriormente, efetuou-se o somatório da pontuação de cada modelo individualmente, sendo selecionados os melhores modelos para cada um dos volumes avaliados. Nos casos de empate no VP, para designar o modelo de desempenho superior, adotou-se como critério de desempate o maior $\mathrm{R}^{2}$ aj.

Os modelos selecionados através dos critérios considerados foram analisados quanto à distribuição gráfica dos resíduos, com finalidade de verificar se havia tendências na estimativa da variável dependente volume, se a variância era homogênea e se o ajuste foi satisfatório ao longo de toda a linha de estimativa. Ao se ajustar um modelo, almeja-se uma distribuição uniforme dos resíduos ao longo de todo o eixo e apresentação de valores de resíduos positivos e negativos (Téo et al., 2011).

\section{Métodos para estimativa do volume}

Para avaliar a eficiência na estimativa do volume total (com e sem casca) de cada árvore foram avaliados três métodos (fator de forma, quociente de forma e modelo volumétrico selecionado em função do melhor desempenho), que foram comparados ao volume obtido pela cubagem rigorosa.

Os volumes individuais obtidos por meio do fator de forma $(f)$, razão entre o volume real e o volume do cilindro com DAP, e do quociente de forma de Schiffel $(Q)$, dado pela fórmula (3), foram calculados com base na média aritmética dos valores individuais de $f$ e de $Q$, a qual foi multiplicada pelo volume do cilindro.

$$
Q=\frac{D_{1} / 2 H}{D A P}, Q<1
$$

em que: $D_{1 / 2 H}=$ diâmetro medido na metade da altura total da árvore. 
Os resultados dos volumes totais com e sem casca foram analisados separadamente, segundo um delineamento inteiramente casualizado com quatro tratamentos (cubagem rigorosa, fator de forma, quociente de forma e modelo volumétrico) e 30 repetições, adotando-se o teste $\mathrm{F}$ a 5\% de significância. Para comparação de médias, utilizou-se o teste de Tukey a 5\% de significância.

\section{Resultados e discussão}

\section{Modelos volumétricos}

Os coeficientes e estatísticas de precisão resultantes do ajuste dos 8 modelos testados para estimativa dos volumes considerados estão apresentados nas Tabelas 3 e 4.

Os valores observados para o parâmetro $R^{2} a j$ estiveram entre $87,9 \%$ e $98,0 \%$, demonstrando alto grau de ajuste da variável dependente em função das variáveis independentes, o que sugere bom desempenho estatístico de todos os modelos testados, tanto para o volume com casca (Tabela 3) quanto para sem casca (Tabela 4). O modelo de Schumacher \& Hall log apresentou os maiores números para esse critério, com valores médios de $97,3 \%$ e $97,9 \%$, respectivamente.

Os modelos testados forneceram baixos valores de $\mathrm{S}_{\mathrm{yx}}$, entre $0,0058 \mathrm{~m}^{3}$ e $0,0167 \mathrm{~m}^{3}$, verificando-se uma maior eficiência dos modelos para os volumes sem casca em comparação aos volumes com casca. Os menores valores foram observados nos modelos Näslund modificado e Stoate para volume sem casca comercial. Resultados semelhantes foram descritos por Pelissari et al. (2011) e Thomas et al. (2006), que observaram superioridade do modelo de Naslund modificado (5) com base no erro padrão da estimativa para povoamentos de Pinus caribaea var. hondurensis e Pinus taeda.

Em geral, os modelos que apresentaram maiores valores de $\mathrm{R}^{2}$ aj foram os de maior desempenho quanto ao $\mathrm{S}_{\mathrm{yx}}$. Segundo Barros et al. (2002), o coeficiente de determinação e o erro padrão residual normalmente estão correlacionados, onde os tratamentos com piores valores para um são também os de menor desempenho para o outro.

Tabela 3. Coeficientes e parâmetros estatísticos obtidos no ajuste dos modelos para volume com casca, total e comercial, de árvores de Pinus caribaea var. hondurensis.

\begin{tabular}{|c|c|c|c|c|c|c|c|c|c|c|}
\hline Modelo & $\boldsymbol{\beta}_{0}^{(1)}$ & $\beta_{1}$ & $\boldsymbol{\beta}_{2}$ & $\boldsymbol{\beta}_{3}$ & $\boldsymbol{\beta}_{4}$ & $\begin{array}{l}\mathbf{R}_{\text {aj }}^{2} \\
(\%)\end{array}$ & $\begin{array}{c}S_{y x} \\
\left(m^{3}\right)\end{array}$ & $\begin{array}{l}S_{y x} \\
(\%)\end{array}$ & $\mathbf{F}$ & VP \\
\hline \multicolumn{11}{|c|}{ Volume total } \\
\hline Hohenadl-Kren & 0,0115 & $-0,0036$ & 0,0004 & & & 92,7 & 0,0136 & 17,0 & 185,3 & 21 \\
\hline Spurr & 3,1951 & $-0,0007$ & & & & 94,4 & 0,0119 & 14,9 & 491,6 & 12 \\
\hline Schumacher \& Hall log & 10,9972 & 1,8823 & 1,3598 & & & 97,1 & 0,0146 & 18,0 & 492,3 & 14 \\
\hline Stoate & $-0,0365$ & 0,0042 & 0,0001 & 1,1463 & & 94,9 & 0,0114 & 14,3 & 179,6 & 10 \\
\hline Näslund modificado & 1466,28 & $-3,2971$ & 325,962 & 0,5280 & 10,5771 & 94,8 & 0,0115 & 14,4 & 132,0 & 15 \\
\hline Equação linear & $-0,0819$ & 0,0106 & & & & 88,9 & 0,0167 & 21,0 & 234,0 & 28 \\
\hline Koperzky\&Gehrhardt & $-0,0129$ & 0,0003 & & & & 92,7 & 0,0136 & 17,0 & 369,9 & 16 \\
\hline Schumacher \& Hall & $-0,1192$ & 0,0077 & 0,0078 & & & 91,3 & 0,0148 & 18,5 & 153,7 & 28 \\
\hline \multicolumn{11}{|c|}{ Volume comercial } \\
\hline Hohenadl-Kren & 0,0144 & $-0,0042$ & 0,0005 & & & 93,6 & 0,0122 & 16,2 & 213,9 & 19 \\
\hline Spurr & 0,0023 & 2,6014 & & & & 95,5 & 0,0103 & 13,6 & 612,5 & 11 \\
\hline Schumacher \& Hall log & $-11,198$ & 1,9411 & 1,3483 & & & 97,5 & 0,0128 & 16,8 & 561,3 & 14 \\
\hline Stoate & $-0,0316$ & 0,0034 & 0,0001 & 1,2973 & & 95,8 & 0,0099 & 13,1 & 221,1 & 10 \\
\hline Näslund modificado & $-0,0358$ & 0,0003 & 0,0030 & 4,5674 & 6,5889 & 92,7 & 0,0130 & 17,3 & 93,5 & 18 \\
\hline Equação linear & $-0,0803$ & 0,0102 & & & & 89,3 & 0,0157 & 20,9 & 244,1 & 28 \\
\hline Koperzky\&Gehrhardt & 0,3397 & $-0,0010$ & & & & 93,5 & 0,0123 & 16,4 & 416,4 & 16 \\
\hline Schumacher \& Hall & $-0,1160$ & 0,0074 & 0,0075 & & & 91,8 & 0,0138 & 18,4 & 162,6 & 28 \\
\hline
\end{tabular}

(1) $\beta_{\mathrm{n}}=$ coeficientes de regressão; $\mathrm{R}_{\mathrm{aj}}^{2}=$ coeficiente de determinação ajustado; $\mathrm{S}_{\mathrm{yx}}=$ erro padrão da estimativa; $\mathrm{S}_{\mathrm{yx}}(\%)=$ erro padrão da estimativa percentual; $\mathrm{F}=$ valor de $\mathrm{F}$ da análise de variância; $\mathrm{VP}=$ valor ponderado dos escores. 
Tabela 4. Coeficientes e parâmetros estatísticos obtidos no ajuste dos modelos para volume sem casca, total e comercial, de árvores de $P$. caribaea var. hondurensis.

\begin{tabular}{|c|c|c|c|c|c|c|c|c|c|c|}
\hline Modelo & $\boldsymbol{\beta}_{0}^{(1)}$ & $\beta_{1}$ & $\boldsymbol{\beta}_{2}$ & $\boldsymbol{\beta}_{3}$ & $\boldsymbol{\beta}_{4}$ & $\begin{array}{l}\mathbf{R}_{\text {aj }}^{2} \\
(\%)\end{array}$ & $\begin{array}{c}\mathbf{S}_{\mathbf{y x}} \\
\left(\mathbf{m}^{3}\right)\end{array}$ & $\begin{array}{c}S_{y x} \\
(\%)\end{array}$ & $\mathbf{F}$ & $\mathbf{V P}$ \\
\hline \multicolumn{11}{|c|}{ Volume total } \\
\hline Hohenadl-Kren & 0,0030 & $-0,0019$ & 0,0004 & & & 90,1 & 0,0107 & 21,3 & 132,6 & 27 \\
\hline Spurr & 0,0020 & 2,8967 & & & & 95,6 & 0,0072 & 14,3 & 625,8 & 9 \\
\hline Schumacher \& Hall log & $-11,2425$ & 1,8767 & 1,4780 & & & 97,9 & 0,0086 & 16,9 & 660,9 & 9 \\
\hline Stoate & $-0,0263$ & 0,0033 & 6,0402 & 1,9538 & & 96,1 & 0,0067 & 13,4 & 236,7 & 6 \\
\hline Näslund modificado & 0,0280 & 0,0006 & $-0,0142$ & $-3,5377$ & 0,0009 & 93,6 & 0,0128 & 16,0 & 106,6 & 23 \\
\hline Equação linear & $-0,0486$ & 0,0085 & & & & 88,0 & 0,0118 & 23,4 & 214,2 & 28 \\
\hline Koperzky\&Gehrhardt & $-0,0070$ & 0,0003 & & & & 91,9 & 0,0097 & 19,3 & 330,5 & 17 \\
\hline Schumacher \& Hall & $-0,0823$ & 0,0057 & 0,0064 & & & 91,7 & 0,0098 & 19,5 & 161,0 & 23 \\
\hline \multicolumn{11}{|c|}{ Volume comercial } \\
\hline Hohenadl-Kren & 0,0044 & $-0,0023$ & 0,0004 & & & 92,1 & 0,0092 & 19,5 & 168,9 & 25 \\
\hline Spurr & 0,0008 & 2,7803 & & & & 96,2 & 0,0064 & 13,6 & 725,3 & 11 \\
\hline Schumacher \& Hall log & $-11,4895$ & 1,9394 & 1,4864 & & & 98,0 & 0,0079 & 16,4 & 723,6 & 11 \\
\hline Stoate & $-0,0242$ & 0,0030 & 3,4505 & 2,0725 & & 96,6 & 0,0060 & 12,7 & 277,5 & 11 \\
\hline Näslund modificado & 0,0193 & 0,0002 & $-0,0072$ & 6,1123 & 0,0002 & 96,8 & 0,0058 & 12,4 & 218,8 & 9 \\
\hline Equação linear & $-0,0474$ & 0,0081 & & & & 87,9 & 0,0113 & 24,0 & 211,9 & 30 \\
\hline Koperzky\&Gehrhardt & $-0,0077$ & 0,0003 & & & & 92,1 & 0,0091 & 19,4 & 339,8 & 18 \\
\hline Schumacher \& Hall & $-0,0804$ & 0,0053 & 0,0062 & & & 91,8 & 0,0093 & 19,8 & 162,8 & 29 \\
\hline
\end{tabular}

(1) $\beta_{\mathrm{n}}=$ coeficientes de regressão; $\mathrm{R}_{\mathrm{aj}}^{2}=$ coeficiente de determinação ajustado; $\mathrm{S}_{\mathrm{yx}}=$ erro padrão da estimativa; $\mathrm{S}_{\mathrm{yx}}(\%)=$ erro padrão da estimativa percentual; $\mathrm{F}=$ valor de $\mathrm{F}$ da análise de variância; $\mathrm{VP}=$ valor ponderado dos escores.

Os resultados referentes ao erro padrão da estimativa percentual $\left(\mathrm{S}_{\mathrm{yx}} \%\right)$ mostraram um melhor desempenho dos modelos para a estimativa dos volumes comerciais. Para estes, observaram-se menores valores nos modelos de Stoate e de Näslund modificado (12,39\% e 13,15\%, respectivamente). Os números encontrados no presente trabalho evidenciam uma baixa variabilidade dos dados, comprovando adequada capacidade para extrapolação dos resultados a partir dos referidos modelos (Silva et al., 2011).

Com relação à estatística $\mathrm{F}$, todos os modelos testados apresentaram valores significativos, demonstrando que os mesmos podem explicar elevadas proporções da variabilidade observada no volume das árvores-amostras. Para os volumes totais, os valores mais significativos de F foram encontrados no modelo Schumacher \& Hall log (média de 576,71), enquanto para o volumes comerciais os maiores valores foram observados no modelo de Spurr (média de 668,88). Silva et. al. (2011) também encontraram resultados superiores de $\mathrm{F}$ para o modelo de Spurr ao estudar equações volumétricas para espécies florestais nativas de valor comercial em Paragominas, PA.
De acordo com o valor ponderado (VP), os modelos de Stoate e de Spurr foram os mais satisfatórios para estimar os volumes com casca. Os modelos de Stoate e de Schumacher \& Hall log foram os mais eficientes para os volume sem casca total, enquanto os modelos de Nalund modificado e de Schumacher \& Hall log mostraram-se os mais adequados para o volume sem casca comercial. Azevedo et al. (2011), estudando povoamentos de Eucalyptus urophylla no Sudoeste da Bahia, encontraram menores valores ponderados para os modelos Schumacher \& Hall log e Spurr.

Também com base no VP, Thomas et al. (2006) observaram superioridade do modelo de Schumacher \& Hall log em Pinus taeda em Santa Catarina, enquanto Pelissari et al. (2011) constataram que os modelos de Naslund modificado e de Spurr foram os mais eficientes para a estimativa de volume de Pinus caribaea var. hondurensis e Pinus tecunumanii em Rondônia. Téo et al. (2011) observaram melhores resultados dos modelos de Stoate e Spurr em povoamentos de Pinus elliottii e Pinus taeda na região de Caçador, SC. 
As Figuras 1 e 2 ilustram a distribuição gráfica dos volumes observados versus volumes estimados pelos dois modelos selecionados para cada um dos volumes considerados, com base no VP.

De um modo geral, a distribuição gráfica dos volumes observados versus estimados pelos modelos selecionados não demonstrou grandes tendências, o que sugere que as estatísticas usadas nos testes de validação podem proporcionar uma seleção adequada de modelos apropriados para a estimativa da variável volume em função da altura e DAP. No entanto, pôde-se verificar pequenas diferenças nas distribuições dos modelos.

Para o volume com casca total e comercial (Figura 1), verifica-se proximidade entre os volumes observados e estimados pelos dois modelos analisados, embora o modelo de Spurr tenha apresentado dispersão levemente superior dos volumes em relação à linha de tendência, o que sugere um melhor desempenho do modelo de Stoate.

Também é possível notar semelhança entre os modelos selecionados para o volume sem casca (Figura 2), que representaram bem a distribuição real dos volumes. Todavia, também é possível observar que, em geral, os modelos de Stoate, para o volume total, e de Naslund, para o volume comercial, geraram volumes mais bem aderidos à linha de tendência, o que sugere que esses modelos seriam os mais satisfatórios. Pelissari et. al. (2011) também verificaram maior eficiência do modelo de Naslund modificado, em relação ao modelo de Schumacher \& Hall log, para estimar o volume de Pinus caribaea e de Pinus tecunumanii.
(A)

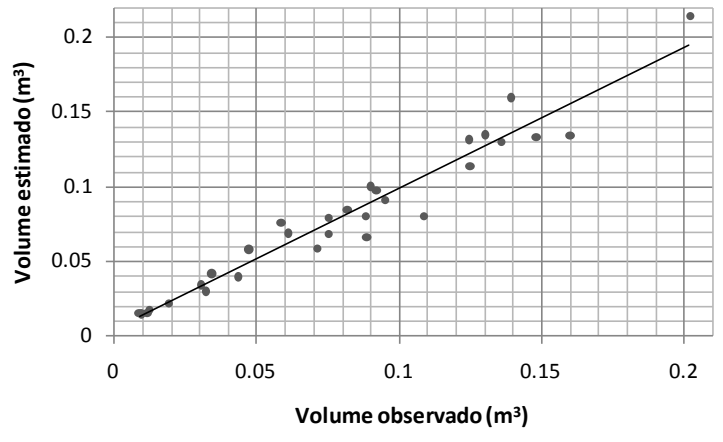

(C)

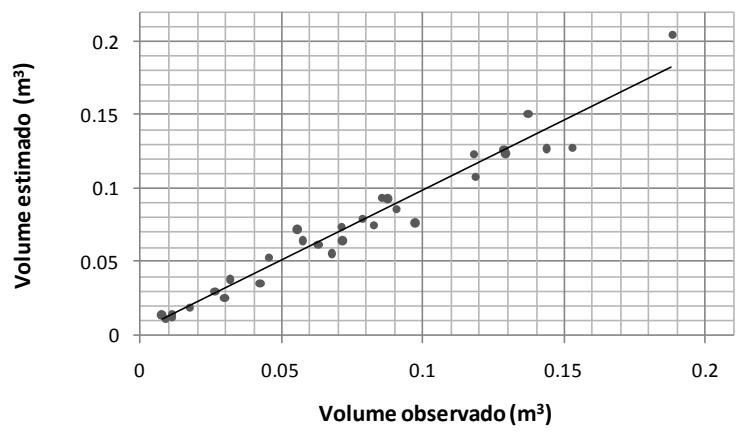

(B)

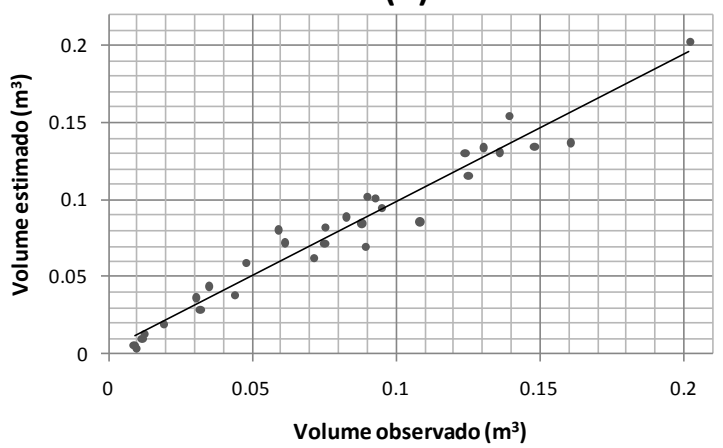

(D)

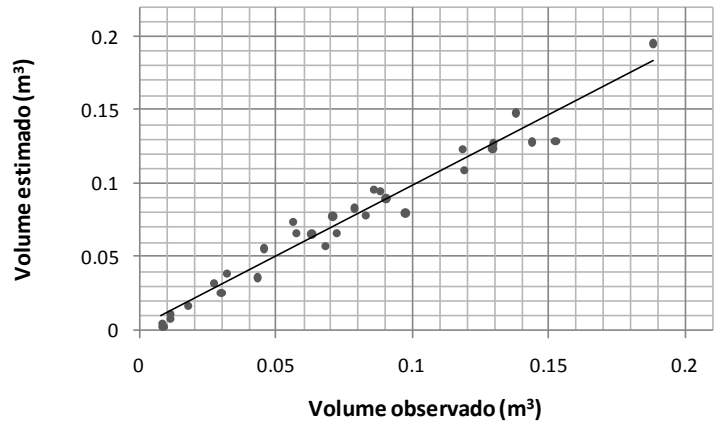

Figura 1. Volumes com casca observados e estimados para Pinus caribaea var. hondurensis a partir dos modelos de Spurr (A) e Stoate (B), para volume total, e dos modelos de Spurr (C) e Stoate (D), para volume comercial. 
(A)

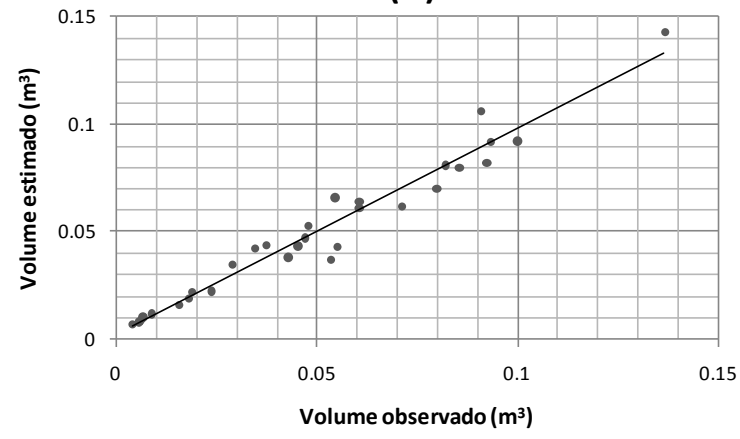

(C)

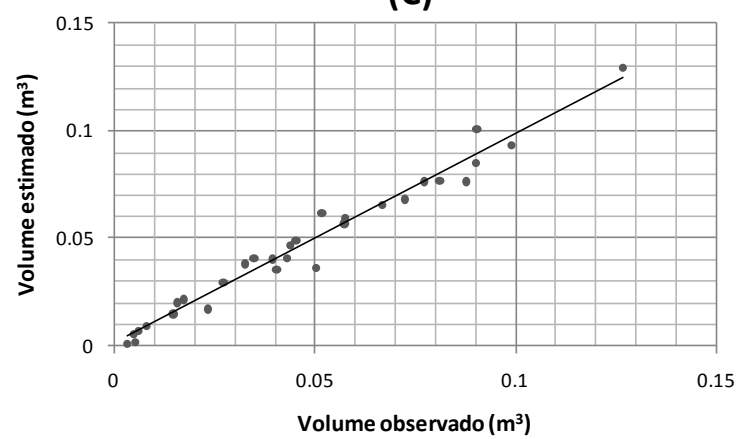

(B)

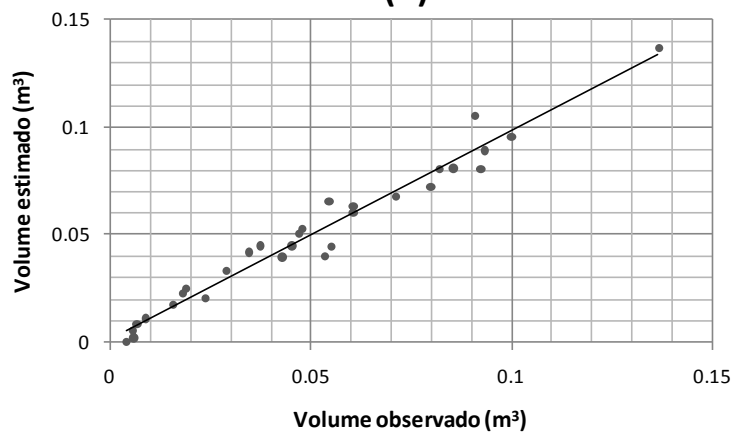

(D)

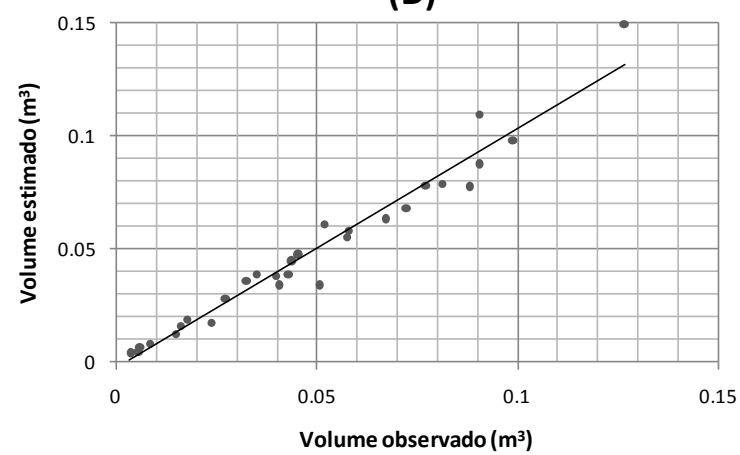

Figura 2. Volumes sem casca observados e estimados para Pinus caribaea var. hondurensis a partir dos modelos de Schumacher \& Hall $\log (\mathrm{A})$ e Stoate (B), para volume total, e dos modelos de Naslund modificado (C) e Schumacher \& Hall log (D), para volume comercial.

\section{Comparação de métodos para estimativa do volume total}

Os valores médios do volume real, obtido com a cubagem rigorosa, e dos volumes totais estimados pelos três métodos considerados (fator de forma, quociente de forma e modelo de maior desempenho) encontram-se apresentados na Tabela 4 . O fator e o quociente de forma médio foram de 0,38 e 0,73 , respectivamente.

Tanto para o volume com casca quanto para o volume sem casca, o valor obtido por meio do quociente de forma foi significativamente maior que os obtidos pelos demais métodos, superestimando os volumes em 46\% a 54\%. Azevedo et al. (2011) também constataram superestimação da variável volume através do quociente de forma de Schiffel.

Os volumes estimados a partir do fator de forma e do emprego do modelo ajustado selecionado apresentaram valores estatisticamente semelhantes entre si e em relação aos volumes obtidos na cubagem, o que evidencia que estes dois métodos foram eficientes na estimativa dos volumes propostos. $\mathrm{Na}$ prática, por questões de facilidade operacional, a comprovada eficiência do fator de forma para a referida espécie pode representar a obtenção de estimativas com precisão em menor tempo e com maior simplicidade de execução.

Tabela 4. Estimativas médias de volume total individual, obtidas por diferentes métodos, para árvores com e sem casca de $P$. caribaea var. hondurensis.

\begin{tabular}{ccccc}
\hline Volume & $\mathbf{V}(\mathbf{C})^{(1)}$ & $\mathbf{V}(\boldsymbol{f})$ & $\mathbf{V}(\boldsymbol{Q})$ & $\mathbf{V}(\mathbf{m o d e l o})$ \\
\hline $\mathbf{C} / \mathbf{C}$ & $0,0799 \mathrm{~b}^{(2)}$ & $0,0799 \mathrm{~b}$ & $0,1477 \mathrm{a}$ & $0,0799 \mathrm{~b}$ \\
$\mathbf{S} / \mathbf{C}$ & $0,0502 \mathrm{~b}$ & $0,0498 \mathrm{~b}$ & $0,1079 \mathrm{a}$ & $0,0506 \mathrm{~b}$ \\
\hline
\end{tabular}

(1) $\mathrm{V}(\mathrm{C})$ = volume médio da cubagem rigorosa; $\mathrm{V}(f)=$ volume médio estimado em função do fator de forma; $\mathrm{V}(Q)=\mathrm{volume}$ médio estimado em função do quociente de forma; V(modelo) = volume médio estimado em função do modelo de melhor desempenho: Stoate, para volume total com casca, e Schumacher \& Hall logaritimizado, para volume total sem casca; ${ }^{(2)}$ Médias seguidas pela mesma letra na linha não diferem entre si pelo teste de Tukey a $5 \%$ de significância. 


\section{Conclusão}

O modelo de Stoate é recomendado para a estimativa dos volumes total e comercial com casca.

Os modelos de Schumacher \& Hall log e de Naslund modificado são indicados para estimar o volume sem casca total e comercial, respectivamente.

$\mathrm{O}$ fator de forma e os modelos volumétricos mostramse adequados para a predição com precisão do volume de madeira de Pinus caribaea var. hondurensis no sudoeste da Bahia.

\section{Referências}

ANUÁRIO estatístico da ABRAF: ano base 2011. Brasília, DF: ABRAF, 2012. 150 p.

AZEVEDo, G. B.; SOUSA, G. T. O.; BARRETO, P. A. B.; CONCEIÇÃO JUNIOR, V. Estimativas volumétricas em povoamentos de eucalipto sob regime de alto fuste e talhadia no sudoeste da Bahia. Pesquisa Florestal Brasileira, Colombo, v. 31, n. 68 , p. 309-318, 2011. DOI: 10.4336/2011.pfb.31.68.309

BARROS, D. A.; MACHADO, S. A.; ACERBI JUNIOR, F. W.; SCOLFORO, J. R. S. Comportamento de modelos hipsométricos tradicionais e genéricos para plantações de Pinus oocarpa em diferentes tratamentos. Boletim Pesquisa Florestal, Colombo, n. 45 , p. $3-28,2002$

BRACELPA. Dados do setor. São Paulo, 2013. Disponível em: $<$ http://www.bracelpa.org.br/bra2/sites/default/files/estatisticas/ booklet.pdf>. Acesso em: 10 out. 2012.

FERRARI, M. P.; FERREIRA, C. A.; SILVA, H. D.; TREVISAN, R. Prognose do crescimento volumétrico individual de árvores de Eucalyptus, em povoamentos na Região Centro Sul. Boletim Pesquisa Florestal, Colombo, n. 51, p. 05-16, 2005.

MACHADO, S. A.; CONCEIÇÃO, M. B.; FIGUEIREDO, D. J. Modelagem do volume individual para diferentes idades e regimes de desbaste em plantações de Pinus oocarpa. Revista Ciências Exatas e Naturais, Guarapuava, v. 4, n. 2, p. 185-197, 2002.

MACHADO, S. A.; URBANO, E.; CONCEIÇÃO, M. B. Comparação de métodos de estimativa de volume para Pinus oocarpa em diferentes idades e diferentes regimes de desbastes. Boletim Pesquisa Florestal, Colombo, n. 50, p. 81-98, 2005.

MORAES NETO, S. P. de Estimativas de volume de Pinus tecunumanii no Cerrado do Distrito Federal. Planaltina, DF: Embrapa Cerrados, 2009. 16 p. (Embrapa Cerrados. Boletim de Pesquisa e Desenvolvimento, 230).

PELISSARI, A. L.; LANSSANOVA, L. R.; DRESCHER, R. Modelos volumétricos para pinus tropicais, em povoamento homogêneo, no Estado de Rondônia. Pesquisa Florestal Brasileira, Colombo, v. 31 , n. 67 , p. $173-181,2011$

RIOS, P. D. A.; MORI, F. A. Estimativa de idade das árvores, massa específica e cubagem da casca de Kielmeyera coriacea Mart. Scientia Forestalis, Piracicaba, v. 40, n. 93, p. 85-93, 2002.

SUPERINTENDÊNCIA DE ESTUDOS ECONÔMICOS E SOCIAIS DA BAHIA. Disponível em: <HTTP://www.sei.ba.gov. br>. Acesso em: 18 set. 2012.

SHIMIZU, J. Y.; MEDRADO, M. J. S. (Ed.). Sistemas de produção: cultivo do pinus. Brasília, DF, 2005. Disponível em: $<$ http://sistemasdeproducao.cnptia.embrapa.br/FontesHTML/Pinus/ CultivodoPinus/apresentacao.htm>, Acesso em: 24 out. 2012.

SHIMIZU, J. Y. Pinus na silvicultura brasileira. Revista da Madeira, Curitiba, v. 16, n. 99, p. 4-14, 2006.

SILVA, E. N.; SANTANA, A. C.; QUEIROZ, W. T.; SOUSA, R. J. Estimação de equações volumétricas para árvores de valor comercial em Paragominas, Estado do Pará. Amazônia: Ciência e Desenvolvimento, Belém, v. 7, n. 13, 2011.

SOARES, C. P. B.; SILVA, G. F.; MARTINS, S. B. Influence of section lenghts on volume determination in Eucalyptus trees. Cerne, Lavras, v. 16, n. 2, p. 155-162, 2010.

TÉO, S. J.; PAZ, R. A.; BORTONCELLO, A. C.; ROCHA, S. P.; COSTA, R. H. Modelagem do volume do povoamento para Pinus taeda L. e Pinus elliottii Engelm., na região de Caçador - SC. Unoesc \& Ciência: ACET, Joaçaba, v. 2, n. 2, p. 193-204, 2011.

THIERSCH, A. A eficiência das distribuições diamétricas para prognose da produção de Eucalyptus camaldulensis. 1997. 155 f. Dissertação (Mestrado em Engenharia Florestal) - Universidade Federal de Lavras, Lavras.

THOMAS, C.; ANDRADE, C. M.; SCHNEIDER, P. R.; FINGER, C. A. G. Comparação de equações volumétricas ajustadas com dados de cubagem e análise de tronco. Ciência Florestal, Santa Maria, RS, v. 16, n. 3, p. 319-327, 2006. 